Mathematical Modelling and Analysis

Volume 21 Number 3, May 2016, 336-349

http://dx.doi.org/10.3846/13926292.2016.1161668

(c) Vilnius Gediminas Technical University, 2016
Publisher: Taylor\&Francis and VGTU

http://www.tandfonline.com/TMMA

ISSN: $1392-6292$

eISSN: $1648-3510$

\title{
Difference Equations in a Multidimensional Space*
}

\author{
Alexander V. Vasilyev ${ }^{a}$ and Vladimir B. Vasilyev ${ }^{b}$ \\ ${ }^{a}$ National Research Belgorod State University \\ Studencheskaya 14/1, 308007 Belgorod, Russia \\ E-mail: alexvassel@gmail.com \\ ${ }^{b}$ Lipetsk State Technical University \\ Moskovskaya 30, 398600 Lipetsk, Russia \\ E-mail(corresp.): vladimir.b.vasilyev@gmail.com
}

Received August 25, 2015; revised February 28, 2016; published online May 15, 2016

\begin{abstract}
One considers a general difference equation in a multidimensional space with continuous coefficients in the space of integrable functions. Necessary and sufficient conditions for a Fredholm property are obtained with a help of the Fourier transform and the Riemann boundary value problem. For simplest cases solvability conditions and formula of a general solution for the difference equation are given.
\end{abstract}

Keywords: difference equation, symbol, singular integral equation, Riemann boundary value problem, index.

AMS Subject Classification: 39A06; 35S05.

\section{Introduction}

We consider a general difference equation in a multidimensional space of the following type

$$
\sum_{|k|=0}^{+\infty} a_{k}(x) u\left(x+\beta_{k}\right)=v(x), \quad x \in D,
$$

where $D$ is $\mathbb{R}^{m}$ or $\mathbb{R}_{+}^{m}=\left\{x \in \mathbb{R}^{m}: x=\left(x_{1}, \cdots, x_{m}\right), x_{m}>0\right\}, k$ is multiindex $k=\left(k_{1}, \cdots, k_{m}\right), \beta_{k}=\left(\beta_{k_{1}}, \cdots, \beta_{k_{m}}\right) \in D$. Our main goal is to describe a Fredholm property for such equations. Such equations have variable coefficients, and only for one-dimensional equations on a straight line with constant coefficients one can construct exact solution [4,6,11]. We'll use methods of the theory of singular integral equations $[2,3,5,7]$, boundary value problems [1], and the Wiener-Hopf technique [8]. Some results related to certain classes of difference equations are described in papers $[12,13,15,16,17]$.

\footnotetext{
* This work was partially supported by Russian Foundation for Basic Research and government of Lipetsk region of Russia, project no. 14-41-03595-a
} 
This paper is devoted to equations with a continual variable. The case of a discrete variable will be considered in a separate paper because there are certain principal distinctions.

\section{The Fourier transform and symbol}

If we consider the equation with constant coefficients in the whole space $\mathbb{R}^{m}$

$$
\sum_{|k|=0}^{+\infty} a_{k} u\left(x+\beta_{k}\right)=v(x), \quad x \in \mathbb{R}^{m},
$$

then we can use the Fourier transform

$$
\tilde{u}(\xi)=\int_{\mathbb{R}^{m}} e^{i x \cdot \xi} u(x) d x
$$

and obtain an equivalent equation in the space $L_{2}\left(\mathbb{R}^{m}\right)$

$$
\sigma(\xi) \tilde{u}(\xi)=\tilde{v}(\xi)
$$

where

$$
\sigma(\xi)=\sum_{k=0}^{+\infty} a_{k} e^{i \beta_{k} \cdot \xi}, \quad \xi \in \mathbb{R}^{m} .
$$

It implies necessary and sufficient condition for a unique solvability of the equation (2.1): if $\sigma \in L_{\infty}\left(\mathbb{R}^{m}\right)$ then this is

$$
\underset{\xi \in \mathbb{R}^{m}}{\operatorname{essinf}}|\sigma(\xi)|>0
$$

Unfortunately we can't use this approach if we are in the space $\mathbb{R}_{+}^{m}$ and consider the equation (1.1) with constant coefficients

$$
\sum_{|k|=0}^{+\infty} a_{k} u\left(x+\beta_{k}\right)=v(x), \quad x \in \mathbb{R}_{+}^{m},
$$

because we have no description for Fourier image of the space $L_{2}\left(\mathbb{R}_{+}^{m}\right)$. Hence the first step is to obtain such description.

This description is a very simple and it is described in [1]. The book [1] is devoted to constructing theory of pseudo differential equations on manifolds with a smooth boundary but methods introduced in the book are applicable to our situation also. Here we'll give a brief sketch of main ideas from [1].

If $u \in L_{2}\left(\mathbb{R}^{m}\right)$ then

$$
F P_{ \pm} u=\Pi_{ \pm} F u
$$

where $P_{ \pm}$is the projection operator on $\mathbb{R}_{ \pm}^{m}$, i.e. $\left(P_{ \pm} u\right)(x)=u(x)$ if $x \in \mathbb{R}_{ \pm}^{m}$, and $\left(P_{ \pm} u\right)(x)=0$ otherwise, $\Pi_{ \pm}$is the following operator

$$
\left(\Pi_{ \pm} u\right)(\xi)=\frac{i}{2 \pi} \int_{\tau \rightarrow 0+}^{+\infty} \frac{u\left(\xi^{\prime}, \eta_{m}\right) d \eta_{m}}{\xi_{m} \pm i \tau-\eta_{m}}, \quad \xi^{\prime}=\left(\xi_{1}, \cdots, \xi_{m-1}\right) .
$$


This relation is a very important for constructing a solution of the difference equation (2.3).

Let's introduce the following notations. Let $\mathcal{A}, \mathcal{B}$ be difference operators of the type

$$
(\mathcal{A} u)(x)=\sum_{|k|=0}^{+\infty} a_{k} u\left(x+\alpha_{k}\right), \quad(\mathcal{B} u)(x)=\sum_{|k|=0}^{+\infty} b_{k} u\left(x+\beta_{k}\right), \quad x \in \mathbb{R}^{m} .
$$

The functions

$$
\sigma_{\mathcal{A}}(\xi)=\sum_{k=0}^{+\infty} e^{i \alpha_{k} \cdot \xi}, \quad \sigma_{\mathcal{B}}(\xi)=\sum_{k=0}^{+\infty} e^{i \beta_{k} \cdot \xi}, \quad \xi \in \mathbb{R}^{m}
$$

are called the symbols of the operators $\mathcal{A}, \mathcal{B}$ respectively.

Obviously these operators $\mathcal{A}, \mathcal{B}$ are linear bounded operators $L_{2}\left(\mathbb{R}^{m}\right) \longrightarrow$ $L_{2}\left(\mathbb{R}^{m}\right)$ if $\sigma_{\mathcal{A}}, \sigma_{\mathcal{B}} \in L_{\infty}\left(\mathbb{R}^{m}\right)$.

The equation (2.3) can be written in an operator form as follows

$$
\left(P_{+} \mathcal{A} u\right)(x)=v(x), \quad x \in \mathbb{R}_{+}^{m} .
$$

One can consider also more general equation in the space $L_{2}\left(\mathbb{R}^{m}\right)$

$$
\left(\mathcal{A} P_{+}+\mathcal{B} P_{-}\right) U=V
$$

and conclude that the equation (2.4) is equivalent to the equation (2.5) with $\mathcal{B} \equiv I$, where $I$ is identity operator. This equivalence means that if we know the solution of (2.4) we can write the solution of

$$
\left(\mathcal{A} P_{+}+I P_{-}\right) U=V
$$

and vice versa. That's why we'll consider more general equation (2.5).

After applying the Fourier transform to (2.5) we obtain

$$
\sigma_{\mathcal{A}}(\xi)\left(\Pi_{+} \tilde{U}\right)(\xi)+\sigma_{\mathcal{B}}(\xi)\left(\Pi_{-} \tilde{U}\right)(\xi)=\tilde{V}(\xi)
$$

and the last equation (2.6) is one-dimensional characteristic singular integral equation with the parameter $\xi^{\prime}$. Such conclusion we have because the introduced operators $\Pi_{ \pm}$are connected to the operator (Plemelj-Sokhotskii formulas)

$$
(H u)(\xi)=\frac{1}{\pi i} v \cdot p \cdot \int_{-\infty}^{+\infty} \frac{u\left(\xi^{\prime}, \eta_{m}\right) d \eta_{m}}{\xi_{m}-\eta_{m}}
$$

which is well-known as the Hilbert transform or one-dimensional singular integral operator $[2,3,5,7]$.

\section{Singular integral equation and Riemann boundary value problem}

The equation (2.6) can be rewritten as the Riemann boundary value problem

$$
\begin{array}{r}
\left(\Pi_{+} \tilde{U}\right)\left(\xi^{\prime}, \xi_{m}\right)=-\sigma_{\mathcal{A}}^{-1}\left(\xi^{\prime}, \xi_{m}\right) \sigma_{\mathcal{B}}\left(\xi^{\prime}, \xi_{m}\right)\left(\Pi_{-} \tilde{U}\right)\left(\xi^{\prime}, \xi_{m}\right) \\
+\sigma_{\mathcal{A}}^{-1}\left(\xi^{\prime}, \xi_{m}\right) \tilde{V}\left(\xi^{\prime}, \xi_{m}\right), \quad \xi_{m} \in \mathbb{R},
\end{array}
$$


or as the one-dimensional singular integral equation

$$
\begin{aligned}
& \frac{\sigma_{\mathcal{A}}\left(\xi^{\prime}, \xi_{m}\right)+\sigma_{\mathcal{B}}\left(\xi^{\prime}, \xi_{m}\right)}{2} \tilde{U}\left(\xi^{\prime}, \xi_{m}\right)+\frac{\sigma_{\mathcal{A}}\left(\xi^{\prime}, \xi_{m}\right)-\sigma_{\mathcal{B}}\left(\xi^{\prime}, \xi_{m}\right)}{2} \\
& \quad \times(H \tilde{U})\left(\xi^{\prime}, \xi_{m}\right)=\tilde{V}\left(\xi^{\prime}, \xi_{m}\right), \quad \xi_{m} \in \mathbb{R}
\end{aligned}
$$

with a parameter $\xi^{\prime} \in \mathbb{R}^{m-1}$.

The theory for such equations or equivalently Riemann boundary value problems is well-known $[2,3,5,7]$. The index of such Riemann boundary value problem plays key role in describing construction for a solution. For our case we have only one difficulty related to a parameter $\xi^{\prime}$. We'll give definition for the index in connection with our case.

Let's denote

$$
\sigma\left(\xi^{\prime}, \xi_{m}\right) \equiv-\sigma_{\mathcal{A}}^{-1}\left(\xi^{\prime}, \xi_{m}\right) \sigma_{\mathcal{B}}\left(\xi^{\prime}, \xi_{m}\right)
$$

and suppose that $\sigma \in C\left(\dot{\mathbb{R}}^{m}\right), \dot{\mathbb{R}}^{m}$ is a compactification of $\mathbb{R}^{m}$.

Definition 1. Symbol $\sigma(\xi)$ is called elliptic if $\sigma(\xi) \neq 0, \forall \xi \in \dot{\mathbb{R}}^{m}$.

Fix $\xi^{\prime} \in \mathbb{R}^{m-1}$ and define

$$
æ\left(\xi^{\prime}\right) \equiv \operatorname{Ind} \sigma=\frac{1}{2 \pi} \int_{-\infty}^{+\infty} d \arg \sigma\left(\xi^{\prime}, \xi_{m}\right) .
$$

Remark 1. This index is an integer, and indeed it doesn't depend on $\xi^{\prime}$ if $m \geq 2$ (homotopy property). The case $m=1$ is a very specific one (see [14]). So we have $æ\left(\xi^{\prime}\right)=æ$.

\section{Solvability condition}

Definition 2. Factorization of the elliptic symbol $\sigma(\xi)$ is called its representation in the form

$$
\sigma(\xi)=\sigma_{+}(\xi) \cdot \sigma_{-}(\xi)
$$

where the factors $\sigma_{ \pm}$admit an analytical continuation into complex half-planes $\mathbb{C}_{ \pm}$and $\sigma_{ \pm} \in L_{\infty}(\mathbb{R})$.

Such factorization (in some more general sense) exists for all cases and can be constructed by the Hilbert transform $H[2,3,5,7]$.

Now we are ready to formulate a basic result on unique solvability of the equation (2.4).

Theorem 1. Let $\sigma(\xi)$ be an elliptic symbol. Then for unique solvability of the equation (2.5) in the space $L_{2}\left(\mathbb{R}^{m}\right)$ it is necessary and sufficient $æ=0$.

Proof. We see that our operator (and equation) are one-dimensional one, hence we can use one-dimensional theory (see [14]). According to the needed result the equation $(2.4)$ has a unique solution in $L_{2}\left(\mathbb{R}_{+}^{m}\right)$ if the condition $æ=0$ holds. 
Of course there are another cases when the index is not zero. One-dimensional constructions for such situations are described in [14]. To adapt such constructions to multi-dimensional case we need more large scale of functional spaces than $L_{2}\left(\mathbb{R}_{+}^{m}\right)$.

\section{Mapping properties of difference operators}

Definition 3. The space $H^{s}\left(\mathbb{R}^{m}\right), s \in \mathbb{R}$, consists of (generalized) functions for which the following norm

$$
\|u\|_{s}=\left(\int_{\mathbb{R}^{m}}|\tilde{u}(\xi)|^{2}(1+|\xi|)^{2 s} d \xi\right)^{1 / 2}
$$

is a finite number.

This space is a Hilbert space, and the Schwartz space $S\left(\mathbb{R}^{m}\right)$ consisting of infinitely differentiable rapidly decreasing at infinity functions is dense in $H^{s}\left(\mathbb{R}^{m}\right)[1]$. Obviously $H^{0}\left(\mathbb{R}^{m}\right)=L_{2}\left(\mathbb{R}^{m}\right)$.

Lemma 1. Let $\sigma_{\mathcal{A}} \in L_{\infty}\left(\mathbb{R}^{m}\right)$. Then the operator $\mathcal{A}$ is a linear bounded operator $H^{s}\left(\mathbb{R}^{m}\right) \longrightarrow H^{s}\left(\mathbb{R}^{m}\right)$.

Proof. Indeed, for $u \in S\left(\mathbb{R}^{m}\right)$

$$
\begin{aligned}
& (F \mathcal{A} u)(\xi)=\int_{\mathbb{R}^{m}} e^{i x \cdot \xi}\left(\sum_{|k|=0}^{\infty} a_{k} u\left(x+\alpha_{k}\right)\right) d x \\
& =\sum_{|k|=0}^{\infty} a_{k} \int_{\mathbb{R}^{m}} e^{i x \cdot \xi} u\left(x+\alpha_{k}\right) d x=\sum_{|k|=0}^{\infty} a_{k} e^{i \alpha_{k} \cdot \xi} \tilde{u}(\xi)=\sigma_{\mathcal{A}}(\xi) \cdot \tilde{u}(\xi),
\end{aligned}
$$

under the condition that sum and integral can be re-arranged, it's possible according to our assumptions, and further

$$
\begin{aligned}
\|\mathcal{A} u\|_{s}^{2} & =\int_{\mathbb{R}^{m}}\left|\sigma_{\mathcal{A}}(\xi) \cdot \tilde{u}(\xi)\right|^{2}(1+|\xi|)^{2 s} d \xi \\
& \leq\left(\operatorname{ess~sup}_{\xi \in \mathbb{R}^{m}}\left|\sigma_{\mathcal{A}}(\xi)\right|\right)^{2} \int_{\mathbb{R}^{m}}|\tilde{u}(\xi)|^{2}(1+|\xi|)^{2 s} d \xi=\left\|\sigma_{\mathcal{A}}\right\|_{L_{\infty}}^{2}\|u\|_{s}^{2}
\end{aligned}
$$

Remark 2. It seems that the operator $\mathcal{A}$ is a pseudo differential operator with the symbol $\sigma_{\mathcal{A}}(\xi)$. It is so but the formula

$$
F_{\xi \rightarrow x}^{-1}\left(\sigma_{\mathcal{A}}(\xi) \cdot \tilde{u}(\xi)\right)
$$

will define a very specific integral operator of convolution type with a nonregular kernel

$$
K_{\mathcal{A}}(x)=\sum_{|k|=0}^{\infty} p_{k} \delta\left(x+\alpha_{k}\right)
$$


so that

$$
F_{\xi \rightarrow x}^{-1}\left(\sigma_{\mathcal{A}}(\xi) \cdot \tilde{u}(\xi)\right)=\int_{\mathbb{R}^{m}}\left(\sum_{|k|=0}^{\infty} p_{k} \delta\left(x+\alpha_{k}-y\right)\right) u(y) d y
$$

\section{Non-vanishing indices}

Here we'll apply our one-dimensional results from [14] to obtain solvability picture for a multi-dimensional case.

\subsection{Positive index}

Let $æ \in \mathbb{N}$. First we introduce a function

$$
\omega\left(\xi^{\prime}, \xi_{m}\right)=\left(\frac{\xi_{m}-i\left|\xi^{\prime}\right|}{\xi_{m}+i\left|\xi^{\prime}\right|}\right)^{æ},
$$

which belongs to $L_{\infty}\left(\mathbb{R}^{m}\right)$.

Evidently the functions $z \pm i\left|\xi^{\prime}\right|$ for fixed $\xi^{\prime} \in \mathbb{R}^{m-1}$ are analytical functions in complex half-planes $\mathbb{C}_{ \pm}$. Moreover

$$
\text { Ind } \frac{\xi_{m}-i\left|\xi^{\prime}\right|}{\xi_{m}+i\left|\xi^{\prime}\right|} \equiv \frac{1}{2 \pi} \int_{-\infty}^{+\infty} d \arg \frac{\xi_{m}-i\left|\xi^{\prime}\right|}{\xi_{m}+i\left|\xi^{\prime}\right|}=1 .
$$

According to the index property a function $\omega^{-1}\left(\xi^{\prime}, \xi_{m}\right) \sigma\left(\xi^{\prime}, \xi_{m}\right)$ has a vanishing index, and it can be factorized in a sense of the definition 1 :

$$
\omega^{-1}\left(\xi^{\prime}, \xi_{m}\right) \sigma\left(\xi^{\prime}, \xi_{m}\right)=\sigma_{+}\left(\xi^{\prime}, \xi_{m}\right) \sigma_{-}\left(\xi^{\prime}, \xi_{m}\right),
$$

so we have $\sigma\left(\xi^{\prime}, \xi_{m}\right)=\omega\left(\xi^{\prime}, \xi_{m}\right) \sigma_{+}\left(\xi^{\prime}, \xi_{m}\right) \sigma_{-}\left(\xi^{\prime}, \xi_{m}\right)$, where

$$
\sigma_{ \pm}\left(\xi^{\prime}, \xi_{m}\right)=\exp \left(\Psi^{ \pm}\left(\xi^{\prime}, \xi_{m}\right)\right), \quad \Psi^{ \pm}\left(\xi^{\prime}, \xi_{m}\right)=\frac{1}{2 \pi i} \lim _{\tau \rightarrow 0+} \int_{-\infty}^{+\infty} \frac{\ln \left(\omega^{-1} \sigma\right)\left(\xi, \eta_{m}\right) d \eta_{m}}{\xi_{m} \pm i \tau-\eta_{m}}
$$

Further we'll apply the Wiener-Hopf technique to the equation (2.6) and write it in a convenient form:

$$
\sigma_{\mathcal{A}}(\xi) \tilde{U}_{+}(\xi)+\sigma_{\mathcal{B}}(\xi) \tilde{U}_{-}(\xi)=\tilde{V}(\xi) .
$$

Taking into account our above calculations we have

$$
\tilde{U}_{+}(\xi)+\omega\left(\xi^{\prime}, \xi_{m}\right) \sigma_{+}\left(\xi^{\prime}, \xi_{m}\right) \sigma_{-}\left(\xi^{\prime}, \xi_{m}\right) \tilde{U}_{-}(\xi)=\sigma_{\mathcal{A}}^{-1}\left(\xi^{\prime}, \xi_{m}\right) \tilde{V}(\xi),
$$

or in other words

$$
\sigma_{+}^{-1}\left(\xi^{\prime}, \xi_{m}\right) \tilde{U}_{+}(\xi)+\omega\left(\xi^{\prime}, \xi_{m}\right) \sigma_{-}\left(\xi^{\prime}, \xi_{m}\right) \tilde{U}_{-}(\xi)=\sigma_{+}^{-1}\left(\xi^{\prime}, \xi_{m}\right) \sigma_{\mathcal{A}}^{-1}\left(\xi^{\prime}, \xi_{m}\right) \tilde{V}(\xi) .
$$

Let's introduce the following notations. We denote for shortness

$$
\sigma_{+}^{-1}\left(\xi^{\prime}, \xi_{m}\right) \sigma_{\mathcal{A}}^{-1}\left(\xi^{\prime}, \xi_{m}\right) \tilde{V}(\xi) \equiv \tilde{h}\left(\xi^{\prime}, \xi_{m}\right)
$$


and define the spaces $A\left(\mathbb{R}^{m}\right), B\left(\mathbb{R}^{m}\right)$ as subspaces of functions from $L_{2}\left(\mathbb{R}^{m}\right)$ which admit an analytical continuation on the last variable $\xi_{m}$ under almost all fixed $\xi^{\prime} \in \mathbb{R}^{m-1}$ into complex half-planes $\mathbb{C}_{+}, \mathbb{C}_{-}$respectively so that

$$
A\left(\mathbb{R}^{m}\right) \oplus B\left(\mathbb{R}^{m}\right)=L_{2}\left(\mathbb{R}^{m}\right) \quad \text { for } \quad \text { a.a. } \xi^{\prime} \in \mathbb{R}^{m-1} .
$$

Since $\tilde{h} \in L_{2}\left(\mathbb{R}^{m}\right)$ it can be represented as a sum $\tilde{h}=\tilde{h}_{+}+\tilde{h}_{-}$, where $\tilde{h}_{+} \in A\left(\mathbb{R}^{m}\right), \tilde{h}_{-} \in B\left(\mathbb{R}^{m}\right)$, and $\tilde{h}_{ \pm}=\Pi_{ \pm} \tilde{h}$. Thus we have

$$
\sigma_{+}^{-1}\left(\xi^{\prime}, \xi_{m}\right) \tilde{U}_{+}(\xi)+\omega\left(\xi^{\prime}, \xi_{m}\right) \sigma_{-}\left(\xi^{\prime}, \xi_{m}\right) \tilde{U}_{-}(\xi)=\tilde{h}_{+}+\tilde{h}_{-}
$$

and taking into account a form of the function $\omega\left(\xi^{\prime}, \xi_{m}\right)$ we write

$$
\begin{gathered}
\left(\xi_{m}+i\left|\xi^{\prime}\right|\right)^{\circledast} \sigma_{+}^{-1}\left(\xi^{\prime}, \xi_{m}\right) \tilde{U}_{+}\left(\xi^{\prime}, \xi_{m}\right)+\left(\xi_{m}-i\left|\xi^{\prime}\right|\right)^{æ} \sigma_{-}\left(\xi^{\prime}, \xi_{m}\right) \tilde{U}_{-}\left(\xi^{\prime}, \xi_{m}\right) \\
=\left(\xi_{m}+i\left|\xi^{\prime}\right|\right)^{\circledast} \tilde{h}_{+}\left(\xi^{\prime}, \xi_{m}\right)+\left(\xi_{m}+i\left|\xi^{\prime}\right|\right)^{æ} \tilde{h}_{-}\left(\xi^{\prime}, \xi_{m}\right)
\end{gathered}
$$

and hence

$$
\begin{aligned}
& \left(\xi_{m}+i\left|\xi^{\prime}\right|\right)^{\circledast} \sigma_{+}^{-1}\left(\xi^{\prime}, \xi_{m}\right) \tilde{U}_{+}\left(\xi^{\prime}, \xi_{m}\right)-\left(\xi_{m}+i\left|\xi^{\prime}\right|\right)^{æ} \tilde{h}_{+}\left(\xi^{\prime}, \xi_{m}\right) \\
& \quad=\left(\xi_{m}+i\left|\xi^{\prime}\right|\right)^{\circledast} \tilde{h}_{-}\left(\xi^{\prime}, \xi_{m}\right)-\left(\xi_{m}-i\left|\xi^{\prime}\right|\right)^{\circledast} \sigma_{-}\left(\xi^{\prime}, \xi_{m}\right) \tilde{U}_{-}\left(\xi^{\prime}, \xi_{m}\right) .
\end{aligned}
$$

The left hand side of the equation (6.1) belongs to $A\left(\mathbb{R}^{m}\right)$ and its analytical continuation into upper complex half-plane on the variable $\xi_{m}$ has a pole of order $æ$ at infinity, and the right hand side belongs to the space $B\left(\mathbb{R}^{m}\right)$ and has the same pole. Then taking into account the generalized Liouville theorem $[2,7]$ we conclude that both left had side and right hand side is a polynomial of order no more than $æ-1$. Since we are interested in left hand side we write

$$
\left(\xi_{m}+i\left|\xi^{\prime}\right|\right)^{æ} \sigma_{+}^{-1}\left(\xi^{\prime}, \xi_{m}\right) \tilde{U}_{+}\left(\xi^{\prime}, \xi_{m}\right)-\left(\xi_{m}+i\left|\xi^{\prime}\right|\right)^{æ} \tilde{h}_{+}\left(\xi^{\prime}, \xi_{m}\right)=\sum_{k=0}^{\infty-1} c_{k}\left(\xi^{\prime}\right) \xi_{m}^{k}
$$

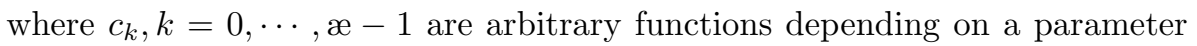
$\xi^{\prime}$. So we have the following formula

$$
\tilde{U}_{+}\left(\xi^{\prime}, \xi_{m}\right)=\sigma_{+}\left(\xi^{\prime}, \xi_{m}\right) \tilde{h}_{+}\left(\xi^{\prime}, \xi_{m}\right)+\left(\xi_{m}+i\left|\xi^{\prime}\right|\right)^{-\circledast} \sigma_{+}\left(\xi^{\prime}, \xi_{m}\right) \sum_{k=0}^{æ-1} c_{k}\left(\xi^{\prime}\right) \xi_{m}^{k}
$$

Obviously the first summand of the last formula belongs to the $L_{2}\left(\mathbb{R}_{+}^{m}\right)$. Since $\sigma_{+} \in L_{\infty}\left(\mathbb{R}^{m}\right)$ then summands $\left(\xi_{m}+i\left|\xi^{\prime}\right|\right)^{-\circledast} c_{k}\left(\xi^{\prime}\right) \xi_{m}^{k}$, should belong to the $L_{2}\left(\mathbb{R}_{+}^{m}\right), k=0, \cdots, æ-1$. It means that

$$
\left.\int_{\mathbb{R}^{m}}\left|\xi_{m}+i\right| \xi^{\prime}\right|^{-2 æ}\left|c_{k}\left(\xi^{\prime}\right)\right|^{2}\left|\xi_{m}\right|^{2 k} d \xi<+\infty .
$$

Passing to a repeated integral we first estimate a one-dimensional integral

$$
\int_{-\infty}^{+\infty}\left|\xi_{m}+i\right| \xi^{\prime}||^{-2 æ}\left|\xi_{m}\right|^{2 k} d \xi_{m}
$$


It exists at infinity only if $-2 æ+2 k<-1$, i.e. $k<æ-1 / 2$.

More precise estimate gives the following for large $\left|\xi^{\prime}\right|$

$$
\int_{-\infty}^{+\infty}\left|\xi_{m}+i\right| \xi^{\prime}||^{-2 æ}\left|\xi_{m}\right|^{2 k} d \xi_{m} \sim \int_{0}^{+\infty}|\xi|^{-2 æ}\left|\xi_{m}\right|^{2 k} d \xi_{m} \sim\left(1+\left|\xi^{\prime}\right|\right)^{-2 æ+2 k+1} .
$$

Returning to the integral (6.2) we see that it exists only if

$$
\int_{\mathbb{R}^{m-1}}\left|c_{k}\left(\xi^{\prime}\right)\right|^{2}\left(1+\left|\xi^{\prime}\right|\right)^{-2 æ+2 k+1} d \xi^{\prime}<+\infty .
$$

The last means that $c_{k} \in H^{s_{k}}\left(\mathbb{R}^{m-1}\right), s_{k}=-\mathfrak{x}+k+1 / 2, k=0, \cdots, \mathfrak{x}-1$.

Thus we have proved the following theorem.

Theorem 2. Let $\sigma(\xi)$ be an elliptic symbol, æ is its index of factorization with factors $\sigma_{ \pm}(\xi)$. If $\in \mathbb{N}$ then the equation (2.5) has many solutions in the space $L_{2}\left(\mathbb{R}^{m}\right)$, and formula for a general solution in Fourier image

$$
\tilde{U}_{+}\left(\xi^{\prime}, \xi_{m}\right)=\sigma_{+}\left(\xi^{\prime}, \xi_{m}\right) \tilde{h}_{+}\left(\xi^{\prime}, \xi_{m}\right)+\left(\xi_{m}+i\left|\xi^{\prime}\right|\right)^{-\circledast} \sigma_{+}\left(\xi^{\prime}, \xi_{m}\right) \sum_{k=0}^{æ-1} c_{k}\left(\xi^{\prime}\right) \xi_{m}^{k}
$$

holds, where lv is an arbitrary continuation $v$ on $\mathbb{R}^{m}, c_{k} \in H^{s_{k}}\left(\mathbb{R}^{m-1}\right), s_{k}=$ $-æ+k+1 / 2, k=0, \cdots, æ-1$, are arbitrary functions.

Corollary 1. If under assumptions of the theorem $3 v \equiv 0$ then a general solution of the equation

$$
(\mathcal{A} u)(x)=0, \quad x \in \mathbb{R}_{+}^{m}
$$

has the form

$$
\tilde{u}(\xi)=\left(\xi_{m}+i\left|\xi^{\prime}\right|\right)^{-\circledast} \sigma_{+}\left(\xi^{\prime}, \xi_{m}\right) \sum_{k=0}^{æ-1} c_{k}\left(\xi^{\prime}\right) \xi_{m}^{k}
$$

Note. If we are interested in the equation (2.4) we need to put $\sigma(\xi)=$ $\sigma_{\mathcal{A}}^{-1}(\xi)$ to obtain this assertion.

\subsection{Negative index}

Theorem 3. Let $\sigma(\xi)$ be an elliptic symbol and $æ<0$. The equation (2.5) has a solution $U \in L_{2}\left(\mathbb{R}^{m}\right)$ iff the right hand side $V$ satisfies the following conditions:

$$
\int_{-\infty}^{+\infty} \frac{\sigma_{+}^{-1}\left(\xi^{\prime}, \eta_{m}\right) \sigma_{\mathcal{A}}^{-1}\left(\xi^{\prime}, \eta_{m}\right) \tilde{V}\left(\xi^{\prime}, \eta_{m}\right) d \eta_{m}}{\left(\eta_{m}+i\left|\xi^{\prime}\right|\right)^{k+1}}=0, \quad k=0,1, \cdots,|æ| .
$$

Proof. Initial point for the proof is the quality (6.1). Then we conclude that both the left hand side and the right hand side are zero because we have a zero of order $-x$ at infinity. Hence

$$
\begin{aligned}
& \left(\xi_{m}+i\left|\xi^{\prime}\right|\right)^{\circledast} \sigma_{+}^{-1}\left(\xi^{\prime}, \xi_{m}\right) \tilde{U}_{+}\left(\xi^{\prime}, \xi_{m}\right)-\left(\xi_{m}+i\left|\xi^{\prime}\right|\right)^{æ} \tilde{h}_{+}\left(\xi^{\prime}, \xi_{m}\right) \\
& \quad=\left(\xi_{m}+i\left|\xi^{\prime}\right|\right)^{æ} \tilde{h}_{-}\left(\xi^{\prime}, \xi_{m}\right)-\left(\xi_{m}-i\left|\xi^{\prime}\right|\right)^{\circledast} \sigma_{-}\left(\xi^{\prime}, \xi_{m}\right) \tilde{U}_{-}\left(\xi^{\prime}, \xi_{m}\right)=0
\end{aligned}
$$


and we have

$$
\begin{aligned}
& \tilde{U}_{+}\left(\xi^{\prime}, \xi_{m}\right)=\sigma_{+}\left(\xi^{\prime}, \xi_{m}\right) \tilde{h}_{+}\left(\xi^{\prime}, \xi_{m}\right), \\
& \tilde{U}_{-}\left(\xi^{\prime}, \xi_{m}\right)=\omega^{-1}\left(\xi^{\prime}, \xi_{m}\right) \sigma_{-}^{-1}\left(\xi^{\prime}, \xi_{m}\right) \tilde{h}_{-}\left(\xi^{\prime}, \xi_{m}\right) \\
& \quad=\left(\frac{\xi_{m}-i\left|\xi^{\prime}\right|}{\xi_{m}+i\left|\xi^{\prime}\right|}\right)^{-\circledast} \sigma_{-}^{-1}\left(\xi^{\prime}, \xi_{m}\right) \tilde{h}_{-}\left(\xi^{\prime}, \xi_{m}\right) .
\end{aligned}
$$

These functions $\tilde{U}_{ \pm}$belong to the spaces $A\left(\mathbb{R}^{m}\right), B\left(\mathbb{R}^{m}\right)$ respectively but the function $\tilde{U}_{-}\left(\xi^{\prime}, z\right)$ has a pole of order $-æ$ in the point $-i\left|\xi^{\prime}\right|$. To exclude such possibility we'll use the expansion of the Cauchy type integral $\tilde{h}_{-}$like [1]:

$\tilde{h}_{-}\left(\xi^{\prime}, \xi_{m}\right)=\left(\Pi_{-} \tilde{h}\right)\left(\xi^{\prime}, \xi_{m}\right)=i \sum_{k=0}^{|æ|} \Lambda_{+}^{k}\left(\xi^{\prime}, \xi_{m}\right) \Pi^{\prime} \Lambda_{+}^{-k-1} \tilde{h}+\Lambda_{+}^{-\infty}\left(\xi^{\prime}, \xi_{m}\right) \Pi_{-} \Lambda_{+}^{æ} \tilde{h}$,

where

$$
\Lambda_{+}\left(\xi^{\prime}, \xi_{m}\right)=\xi_{m}+i\left|\xi^{\prime}\right|, \quad\left(\Pi^{\prime} \tilde{h}\right)\left(\xi^{\prime}\right) \equiv \int_{-\infty}^{+\infty} \tilde{h}\left(\xi^{\prime}, \xi_{m}\right) d \xi_{m}
$$

and notation $\Lambda_{+} \tilde{h}$ denotes a product of two functions $\Lambda_{+}\left(\xi^{\prime}, \xi_{m}\right)$ and $\tilde{h}\left(\xi^{\prime}, \xi_{m}\right)$.

Thus the pole in the point $-i\left|\xi^{\prime}\right|$ will disappear if the following conditions hold:

$$
\int_{-\infty}^{+\infty} \frac{\tilde{h}\left(\xi^{\prime}, \eta_{m}\right) d \eta_{m}}{\left(\eta_{m}+i\left|\xi^{\prime}\right|\right)^{k+1}}=0 \quad \text { for } \quad \text { a.a. } \xi^{\prime} \in \mathbb{R}^{m-1}, \quad k=0,1, \cdots,|æ| .
$$

Taking into account that

$$
\tilde{h}\left(\xi^{\prime}, \xi_{m}\right)=\sigma_{+}^{-1}\left(\xi^{\prime}, \xi_{m}\right) \sigma_{\mathcal{A}}^{-1}\left(\xi^{\prime}, \xi_{m}\right) \tilde{V}(\xi)
$$

and substituting it in obtained conditions we have

$$
\int_{-\infty}^{+\infty} \frac{\sigma_{+}^{-1}\left(\xi^{\prime}, \eta_{m}\right) \sigma_{\mathcal{A}}^{-1}\left(\xi^{\prime}, \eta_{m}\right) \tilde{V}\left(\xi^{\prime}, \eta_{m}\right) d \eta_{m}}{\left(\eta_{m}+i\left|\xi^{\prime}\right|\right)^{k+1}}=0
$$

Remark 3. If we'll enlarge the space scale and consider the Sobolev-Slobodetskii spaces $H^{s}$ then we'll can omit these conditions. But since this problem will be over-determined we need some additional unknowns. These are so-called coboundary operators or potential like operators [1]. We'll study these situations in a separate paper.

\section{$7 \quad$ Variable coefficients}

Here we consider a difference operator with variable coefficients in the space $L_{2}\left(\mathbb{R}^{m}\right)$ :

$$
(\mathcal{D} u)(x)=\sum_{|k|=0}^{+\infty} p_{k}(x) u\left(x+\gamma_{k}\right), \quad x \in \mathbb{R}^{m}, \quad \gamma_{k}=\left(\gamma_{k_{1}}, \cdots, \gamma_{k_{m}}\right) \in \mathbb{R}^{m} .
$$


DEFinition 4. The function

$$
\sigma_{\mathcal{D}}(x, \xi)=\sum_{|k|=0}^{+\infty} p_{k}(x) e^{i \gamma_{k} \cdot \xi}
$$

is called a symbol of the operator $\mathcal{D}$.

We'll suppose in this section that all functions $p_{k}(x),|k|=0,1, \cdots$ are continuous in $\mathbb{R}^{m}$, denote

$$
\left\|p_{k}\right\|_{C\left(\mathbb{R}^{m}\right)}=p_{k},
$$

assuming

$$
\sum_{|k|=0}^{+\infty} p_{k}=A<+\infty
$$

Lemma 2. The operator $\mathcal{D}$ is a linear bounded operator $L_{p}\left(\mathbb{R}^{m}\right) \longrightarrow: L_{p}\left(\mathbb{R}^{m}\right)$, $1 \leq p \leq+\infty$.

Proof. We have

$$
|(\mathcal{D} u)(x)| \leq \sum_{|k|=0}^{+\infty}\left|p_{k}(x)\right|\left|u\left(x+\gamma_{k}\right)\right| \leq \sum_{k=0}^{+\infty} p_{k}\left|u\left(x+\gamma_{k}\right)\right|
$$

and then

$$
\|\mathcal{D} u\|_{1}=\int_{\mathbb{R}^{m}}|(\mathcal{D} u)(x)| d x \leq \sum_{k=0}^{+\infty} p_{k} \int_{\mathbb{R}^{m}}\left|u\left(x+\gamma_{k}\right)\right| \leq A\|u\|_{1},
$$

i.e. $\mathcal{D}: L_{1}\left(\mathbb{R}^{m}\right) \longrightarrow: L_{1}\left(\mathbb{R}^{m}\right)$ is a linear bounded operator.

In the same way we conclude that $\mathcal{D}: L_{\infty}\left(\mathbb{R}^{m}\right) \longrightarrow: L_{\infty}\left(\mathbb{R}^{m}\right)$. Further we can apply simplest interpolation theorems $[10,18]$ and conclude that the operator $\mathcal{D}: L_{p}\left(\mathbb{R}^{m}\right) \longrightarrow: L_{p}\left(\mathbb{R}^{m}\right)$ is a linear bounded operator for all $1 \leq p \leq$ $+\infty$.

\section{$7.1 \quad$ Local principle}

For every operator $\mathcal{D}$ we define an operator family $\left\{\mathcal{D}_{x_{0}}\right\}_{x_{0} \in \dot{\mathbb{R}}^{m}}$ where

$$
\mathcal{D}_{x_{0}}: u(x) \longrightarrow \sum_{|k|=0}^{+\infty} p_{k}\left(x_{0}\right) u\left(x+\gamma_{k}\right), \quad x \in \mathbb{R}^{m}
$$

and the function

$$
\sigma\left(x_{0}, \xi\right)=\sum_{|k|=0}^{+\infty} p_{k}\left(x_{0}\right) e^{i \gamma_{k} \cdot \xi}
$$

will be its symbol (see $(2.2)$ ) because the operator $\mathcal{D}_{x_{0}}$ is an operator with constant coefficients. Following Simonenko [9] we call the operator $\mathcal{D}_{x_{0}}$ a local representative of the operator $\mathcal{D}$ in the point $x_{0}$. 
Let $\mathfrak{L}(\mathcal{D})$ be a space of linear bounded operators of type (7.1) with operator norm $\|\mathcal{D}\|_{L_{p}\left(\mathbb{R}^{m}\right) \rightarrow L_{p}\left(\mathbb{R}^{m}\right)}, 1 \leq p \leq+\infty$. Thus we can introduce the operator function $\mathcal{D}(x) \equiv \mathcal{D}_{x}$ for all $x \in \dot{\mathbb{R}}^{m}$.

We remind that an operator $\mathcal{D}$ has a Fredholm property if

$$
\text { Ind } A \equiv \operatorname{dim} \text { Ker } A-\operatorname{dim} \text { Coker } A
$$

is a finite number. A Fredholm property is stable under compact and small perturbations, and Ind $A$ is a homotopic invariant of the operator $A$ [5].

Lemma 3. Operator $\mathcal{D}$ has a Fredholm property in the space $L_{p}\left(\mathbb{R}^{m}\right)$ iff the family $\left\{\mathcal{D}_{x_{0}}\right\}_{x_{0} \in \mathbb{R}^{m}}$ consists of invertible operators.

Proof. Let $\mathfrak{D}$ be an algebra of difference operators of type (7.1), and $\mathfrak{P}$ be an algebra of pseudo differential operators with symbols $p(x, \xi) \in C\left(\dot{\mathbb{R}}^{m} \times \dot{\mathbb{R}}^{m}\right)$. Obviously $\mathfrak{D} \subset \mathfrak{P}$. Further let $\mathcal{P}(x)$ be a corresponding operator function for a pseudo differential operator $\mathcal{P} \in \mathfrak{P}$. We say that operator function is invertible if exists an operator function $\mathcal{P}^{-1}(x)$ such that

$$
\mathcal{P}(x) \mathcal{P}^{-1}(x)=I, \quad \forall x \in \dot{\mathbb{R}}^{m} .
$$

Now our main goal is to show that given operator function $\mathcal{D}(x)$ one can construct a pseudo differential operator $\mathcal{P} \in \mathfrak{P}$ such that $\mathcal{D} \mathcal{P}$ and $\mathcal{P} \mathcal{D}$ can be represented in the form $I+T$, where $T$ is a compact operator. It will imply that the operator $\mathcal{D}$ has a two-sided regularizer, and thus will have a Fredholm property [5].

In the algebra $\mathfrak{P}$ we'll extract a special type of operators so called operators of a local type [9]. Without going into details let's say that pseudo differential operator from $\mathcal{P} \in \mathfrak{P}$ will be an operator of a local type if its kernel

$$
K_{\mathcal{P}}(x, y)=F_{\xi \rightarrow y}^{-1} p(x, \xi)
$$

is at least a continual function of variables $(x, y)$ and generates a linear bounded operator of the type

$$
u(x) \longmapsto \int_{\mathbb{R}^{m}} K_{\mathcal{P}}(x, x-y) u(y) d y .
$$

Further we'll correct a symbol $p(x, \xi) \in C\left(\dot{\mathbb{R}}^{m} \times \dot{\mathbb{R}}^{m}\right)$ by small perturbations so that the corrected pseudo differential operator $\mathcal{P}_{\varepsilon}$ with a symbol $p_{\varepsilon}(x, \xi)$ will generate the continual kernel $K_{\mathcal{P}, \varepsilon}(x, \xi)$. We need two steps.

First if $p(\infty)=c$ then we consider

$$
p_{1}(x, \xi)=p(x, \xi)-c,
$$

so that $p_{1}(\infty)=0$, and $p(x, \xi)=p_{1}(x, \xi)+c$. It means that the operator with the symbol $p(x, \xi)$ is represented in the form $\mathcal{P}_{1}+c I$.

Second one can approximate the symbol $p_{1}(x, \xi)$ by symbols $p_{1, \varepsilon}(x, \xi) \in$ $S\left(\mathbb{R}^{m} \times \mathbb{R}^{m}\right)$ (Schwartz class) with smooth kernel $K_{\mathcal{P}_{1, \varepsilon}}(x, y)$. Such operator

$$
\left(\mathcal{P}_{\varepsilon} u\right)(x)=c u(x)+\int_{\mathbb{R}^{m}} K_{\mathcal{P}_{1, \varepsilon}}(x, x-y) u(y) d y
$$


is an operator of a local type, and

$$
\left\|\mathcal{P}-\mathcal{P}_{\varepsilon}\right\|_{L_{p}\left(\mathbb{R}^{m}\right) \longrightarrow: L_{p}\left(\mathbb{R}^{m}\right)} \leq \varepsilon .
$$

It's well known $[5,9]$ that the family $\left\{\mathcal{P}_{\varepsilon}(x)\right\}$ of operators of a local type reconstructs the operator $\mathcal{P}_{\varepsilon}$ up to compact summand. Moreover now we can assert the following. If the operator function $\mathcal{P}_{\varepsilon}(x)$ is invertible then the operator $\mathcal{P}_{\varepsilon}^{-1}$ with the symbol $p_{\varepsilon}^{-1}(x, \xi)$ will be two-sided regularizer for the operator $\mathcal{P}_{\varepsilon}$ and consequently for $\mathcal{P}$.

Corollary 2. Operator $\mathcal{D}: L_{p}\left(\mathbb{R}^{m}\right) \rightarrow L_{p}\left(\mathbb{R}^{m}\right)$ has a Fredholm property iff

$$
\sigma_{\mathcal{D}}(x, \xi) \neq 0, \quad \forall x, \xi \in \dot{\mathbb{R}}^{m} .
$$

Lemma 4. Index of a Fredholm operator $\mathcal{D}: L_{p}\left(\mathbb{R}^{m}\right) \rightarrow L_{p}\left(\mathbb{R}^{m}\right)$ is equal to 0 .

Proof. According to convexity of $\dot{\mathbb{R}}^{m}$ one can easily construct a homotopy

$$
\mathcal{D}_{t, x_{0}} \equiv \mathcal{D}_{(1-t) x_{0}+t x}, \quad t \in[0,1]
$$

where $x_{0} \in \dot{\mathbb{R}}^{m}$ is an arbitrary fixed point. Since all intermediate operators are invertible then an initial operator $\mathcal{D}=\mathcal{D}_{1, x_{0}}$ has vanishing index.

\subsection{Half-space case}

This section is connected to operators in $\mathcal{D}: L_{2}\left(\mathbb{R}_{+}^{m}\right) \rightarrow L_{2}\left(\mathbb{R}_{+}^{m}\right)$. These construction are more complicated but a general idea is the same. The main result for this case is the following.

Theorem 4. Operator $\mathcal{D}: L_{2}\left(\mathbb{R}_{+}^{m}\right) \rightarrow L_{2}\left(\mathbb{R}_{+}^{m}\right)$ has a Fredholm property iff the following conditions

$$
\text { 1) } \left.\sigma_{\mathcal{D}}(x, \xi) \neq 0, \quad \forall x \in \overline{\mathbb{R}_{+}^{m}}, \quad \xi \in \dot{\mathbb{R}}^{m}, \quad 2\right) \int_{-\infty}^{+\infty} d \arg \sigma_{\mathcal{D}}\left(\cdot, \cdot \cdot \xi_{m}\right)=0 .
$$

hold.

Sketch of proof. In this case also a local principle plays key role. But main lemma describing the local principle will be distinct. Namely for our difference operator $\mathcal{D}$ the operator function will consist of two parts. The first part $\mathcal{D}_{1}(x)$ will be defined on $\overline{\mathbb{R}_{+}^{m}}$ and it generated by operators of type (7.2); more precisely it consists of pseudo differential operators with symbols $\sigma_{\mathcal{D}}(x, \xi$ :

$$
u(x) \longmapsto F_{\xi \rightarrow x}^{-1} \sigma_{\mathcal{D}}(x, \xi) \tilde{u}(\xi) .
$$

The second part $\mathcal{D}_{2}(x)$ consists of operators of the following type

$$
u(x) \longmapsto \int_{\mathbb{R}_{+}^{m}} K_{\mathcal{D}}(x, x-y) u(y) d y,
$$


or in more general form and in Fourier image

$$
\tilde{u}(\xi) \longmapsto \sigma_{\mathcal{A}}(x, \xi)\left(\Pi_{+} \tilde{u}\right)(x, \xi)+\sigma_{\mathcal{B}}(x, \xi)\left(\Pi_{-} \tilde{u}\right)(x, \xi) .
$$

(We'll remind here that for the operator $\mathcal{D}: L_{2}\left(\mathbb{R}_{+}^{m}\right) \rightarrow L_{2}\left(\mathbb{R}_{+}^{m}\right)$ one should put in the last formula $\left.\sigma_{\mathcal{A}}(x, \xi)=\sigma_{\mathcal{D}}(x, \xi), \sigma_{\mathcal{B}}(x, \xi)=1\right)$. The operator function $\mathcal{D}_{2}(x)$ will be defined for the points $x \in \dot{\mathbb{R}}^{m-1}$, i.e for boundary points of $\mathbb{R}_{+}^{m}$. Further we should prove that operator $\mathcal{D}$ has a Fredholm property iff both parts $\mathcal{D}_{1}(x)$ and $\mathcal{D}_{2}(x)$ are invertible for all admissible $x$. If so then the condition 1 in the theorem is an invertibility condition for the first operator family, and the condition 2 is the same for the second part.

\section{Conclusions}

There are many other interesting cases for studying solvability for difference equations in special canonical domain like multidimensional cones. This will be the object of another paper.

\section{Acknowledgements}

We would like to thank referees for their helpful remarks.

\section{References}

[1] G. Eskin. Boundary value problems for elliptic pseudodifferential equations. AMS, Providence, 1981.

[2] F. D. Gakhov. Boundary value problems. Dover Publications, New York, 1981.

[3] I. Gokhberg and N. Krupnik. Introduction to the theory of one-dimensional singular integral equation. Birkhauser, Basel, 2010.

[4] C. Jordan. Calculus of finite differences. Chelsea Publishing Company, New York, 1950.

[5] S. G. Mikhlin and S. Prössdorf. Singular integral operators. Akademie-Verlag, Berlin, 1986.

[6] L. M. Milne-Thomson. The calculus of finite differences. Chelsea Publishing Company, New York, 1981.

[7] N. I. Muskhelishvili. Singular integral equations. North Holland, Amsterdam, 1976.

[8] B. Noble. Methods based on Wiener-Hopf technique for the solution of partial differential equations. Pergamon Press, London-New York-Paris-Los Angeles, 1958.

[9] I. B. Simonenko. Local method in the theory of shift invariant operators and their envelopings. CVVR, Rostov on Don, 2007. (in Russian)

[10] E. M. Stein and G. Weiss. Introduction to Fourier analysis on Euclidean spaces. Princeton Univ. Press, Princeton, 1971.

[11] E. Titchmarsh. Introduction to the theory of Fourier integrals. Chelsea Publishing Company, New York, 1986. 
[12] A. Vasilyev and V. Vasilyev. Discrete singular operators and equations in a half-space. Azerbaijan Journal of Mathematics, 3(1):84-93, 2013.

[13] A. Vasilyev and V. Vasilyev. Discrete singular integrals in a half-space. In V. Mityushev and M. Ruzhansky(Eds.), Proc. of the 9th ISAAC Congress, Krakow, Poland, 2013, Current Trends in Analysis and Its Applications, Trends in Mathematics, pp. 663-670, Basel, 2015. Birkhauser. http://dx.doi.org/10.1007/9783-319-12577-0.

[14] A. Vasilyev and V. Vasilyev. On some classes of difference equations of infinite order. Advances in Difference Equations, 211:1-9, 2015. http://dx.doi.org/10.1186/s13662-015-0542-3.

[15] A. Vasilyev and V. Vasilyev. Periodic Riemann problem and discrete convolution equations. Differential Equations, 51(5):652-660, 2015. http://dx.doi.org/10.1134/S0012266115050080.

[16] V. Vasilyev. General boundary value problems for pseudo differential equations and related difference equations. Advances in Difference Equations, 289:1-7, 2013. http://dx.doi.org/10.1186/1687-1847-2013-289.

[17] V. Vasilyev. On some difference equations of first order. Tatra Mountains Mathematical Publications, 54:165-181, 2013.

[18] A. Zygmund. Trigonometric series. Cambridge Univ. Press, Cambridge, 1959. 